\title{
Jugendstrafvollzug und Verfassungsrecht
}

\author{
Eine Besprechung des Urteils des BVerfG vom 31.5.2006 zur Verfassungsmäßigkeit des \\ Jugendstrafvollzugs und Folgerungen für die anstehende Gesetzesreform
}

Frieder Dünkel

C ie Notwendigkeit einer gesetzlichen Regelung des Jugendstrafvollzugs ist seit der Einsetzung der Jugendstrafvollzugskommission im Jahre 1976 und der Vorlage des Abschlussberichts der Kommission (1980) bekannt. Da eine Mehrheit der Bundesländer die Reformentwürfe des Bundesjustizministeriums aus den Jahren 1982, 1984, 1991 und 1993 vor allem mit Kostenargumenten immer wieder erfolgreich zu verhindern verstand, blieb es bei den marginalen gesetzlichen Regelungen der $\$ \S 91,92$ JGG, 176, 178 StVollzG. Die Praxis behalf sich mit der weitgehenden Übernahme des StVollzG in den VVJug (Verwaltungsvorschriften zum Jugendstrafvoll$\mathrm{zug})$, die allerdings die Anforderungen an ein förmliches Gesetz nicht erfüllen. Es war daher wenig überraschend, dass das BVerfG im Einklang mit seiner seit 1972 entwickelten Rechtsprechung zum allgemeinen Strafvollzug nunmehr den Verstoß gegen die Verfassung feststellte und dem Gesetzgeber eine Frist bis Ende 2007 setzte, ein förmliches Gesetz zu schaffen, das die Rechte und Pflichten der Jugendstrafgefangenen detailliert regelt. In Anbetracht der Ende Juni 2006 beschlossenen Föderalismusreform und der nunmehr geschaffenen Zuständigkeit der Bundesländer ist das Urteil des BVerfG vor allem deshalb von herausragender Bedeutung, weil es (verfassungsrechtliche) Mindeststandards setzt, die - so bleibt zu hoffen - einem föderalen Flickenteppich unter dem Primat ökonomischer Sparzwänge bzw. „Wettbewerb der Schäbigkeit" (Dünkel in der Anhörung beim BVerfG) entgegen wirken könnte. Der nachfolgende Beitrag gibt die wesentlichen Inhalte des Urteils und die daraus für die Gesetzgebung zu ziehenden Folgerungen wieder.

\section{Das Urteil des BVerfG}

Das BVerfG hat in seiner Entscheidung vom 31.5.2006 (2 BvR 1673/04 - 2 BvR 2402/04, NJW 2006, 2093 ff. = ZJJ 2006, 193 ff. m. Anm. Ostendorf NJW 2006, 2073 f.) entsprechend seiner 1972 ergangenen Entscheidung zum Strafvollzug bei Erwachsenen (vgl. BVerfGE 33, 1) festgestellt, dass auch der Jugendstrafvollzug einer gesetzlichen Grundlage bedarf und die wenigen Vorschriften der §§ 91, 92 JGG, 176, 178 StVollzG keine ausreichende Grundlage für die Einschränkung von Grundrechten der Gefangenen sind.

„Grundrechtseingriffe, die über den Freiheitsentzug als solchen hinausgehen, bedürfen ... einer gesetzlichen Grundlage, die die Eingriffsvoraussetzungen in hinreichend bestimmter Weise normiert (vgl. BVerfGE 40, 276, 283)“ (Rn. 35¹ = NJW 2006, 2094). Insoweit gilt für junge Gefangene nichts anderes als für andere Gefangene. „Hinsichtlich der verfassungsrechtlich gebotenen Regelungsform für Grundrechtseingriffe besteht daher zwischen Erwachsenenvollzug und Jugendstrafvollzug kein Unterschied" (Rn. 36). „Die inhaltliche Ausgestaltung des Strafvollzugs für Jugendliche und ihnen in der Entwicklung gleichstehende heranwachsende Straftäter unterliegt allerdings besonderen verfassungsrechtlichen Anforderungen, die auch für die Reichweite des Erfordernisses gesetzlicher Regelung im Jugendstrafvollzug von Bedeutung sind." (Rn. 37).

Im weiteren führt das BVerfG aus, dass sowohl für die im vorliegenden Fall der Kläger relevanten Vorschriften des StVollzG zur Verhängung von Disziplinarmaßnahmen wie auch bzgl. der Postkontrolle eine analoge Anwendung des StVollzG nicht in Betracht komme. Eine analoge Anwendung der Vorschriften des StVollzG sei schon wegen Art. 103 II GG (das Analogieverbot zuungunsten des Betroffenen gilt auch im Disziplinarrecht) ausgeschlossen. Zudem bestehe hier keine planwidrige Gesetzeslücke, denn der Gesetzgeber des StVollzG wollte bewusst den Jugendstrafvollzug ausklammern und eine eigenständige gesetzliche Regelung schaffen (vgl. Rn. 44 ff. =NJW 2006, 2094 f.).

Ferner fehle es für eine analoge Anwendung des StVollzG an der „erforderlichen Gleichartigkeit der zu regelnden Sachverhalte. Erwachsenen- und Jugendstrafvollzug haben es im Gegenteil mit so unterschiedlichen Sachverhalten zu tun, dass das Strafvollzugsgesetz in seiner geltenden Fassung den verfassungsrechtlichen Anforderungen an eine gesetzliche Regelung ... auch dann nicht entspräche, wenn seine Anwendung für den Jugendstrafvollzug ausdrücklich vorgesehen wäre. Für den Jugendstrafvollzug bedarf es gesetzlicher Grundlagen, die auf die besonderen Anforderungen des Vollzuges von Strafen an Jugendlichen und ihnen gleichstehenden Heranwachsenden zugeschnitten sind" (Rn. 49 = NJW 2006, 2095).

Die Besonderheiten des Jugendstrafvollzugs werden sodann wie folgt begründet: „Die Ausgangsbedingungen und Folgen strafrechtlicher Zurechnung sind bei Jugendlichen in wesentlichen Hinsichten andere als bei Erwachsenen ... Jugendliche befinden sich biologisch, psychisch und sozial in einem Stadium des Übergangs, das typischerweise mit Spannungen, Unsicherheiten und Anpassungsschwierigkeiten, häufig auch in der Aneignung von Verhaltensnormen, verbunden ist. Zudem steht der Jugendliche noch in einem Alter, in dem nicht nur er selbst, sondern auch andere für seine Entwicklung verantwortlich sind. Die Fehlentwicklung, die sich in gravierenden Straftaten eines Jugendlichen äußert, steht in besonders dichtem und oft auch besonders offensichtlichem Zusammenhang mit einem Umfeld und Umständen, die ihn geprägt haben. Für das Jugendstrafrecht und den Jugendstrafvollzug gewinnt daher der Grundsatz, dass Strafe nur letztes Mittel (vgl. BVerfGE 90, 145, 201) und nur als ein in seinen negativen Auswirkungen auf die Persönlichkeit des Betroffenen nach Möglichkeit zu minimierendes Übel (vgl. BVerfGE 45, 187, 238; 64, 261, 272 f.) verhängt und vollzogen werden darf, eine besondere Bedeutung" (Rn. 50).

Weiterhin betont das BVerfG den in ständiger Rspr. entwickelten Verfassungsrang des Resozialisierungsziels auch für den Jugendstrafvollzug. „Zugleich folgt die Notwendigkeit, den Strafvollzug am Ziel der Resozialisierung auszurichten, auch aus der staatlichen Schutzpflicht für die Sicherheit aller Bürger. Zwischen dem Integrationsziel des Vollzugs und dem Anliegen, die Allgemeinheit vor weiteren Straftaten zu schützen, besteht insoweit kein Gegensatz" (Rn. 51).

„Für den Jugendstrafvollzug hat das Ziel der Befähigung $z u$ einem straffreien Leben in Freiheit besonders hohes Gewicht" (Rn. 52). „Dies ergibt sich schon daraus, dass die Verpflichtung des Staates, negative Auswirkungen des Strafübels auf die Lebenstüchtigkeit des Gefangenen weitestgehend zu mindern, hier besonders ausgeprägt ist. Auf den Jugendlichen wirkt die Freiheitsstrafe in einer Lebensphase ein, die auch bei nicht delinquentem Verlauf noch der Entwicklung zu einer Persönlichkeit dient, die in der Lage ist, ein rechtschaffenes Leben in voller Selbständigkeit zu führen. Indem der Staat in diese Lebensphase durch Entzug der Freiheit eingreift, übernimmt er für die weitere Entwicklung des Betroffenen eine besondere Verantwortung. Dieser gesteigerten Verantwortung kann er nur durch eine Vollzugsgestaltung gerecht werden, die in besonderer Weise auf Förderung - vor allem auf soziales Lernen sowie die Ausbildung von Fähigkeiten und Kenntnissen, die einer künftigen beruflichen Integration dienen - gerichtet ist." Da Jugendstrafgefangene in einem statistisch gesehen „kriminalitätsanfälligen Alter" entlassen werden, ist die erfolgreiche Wiedereingliederung „,on besonders großer Bedeu- 
tung“ (Rn. 53). „Freiheitsstrafen wirken sich für Jugendliche in besonders einschneidender Weise aus. Das Zeitempfinden ist anders als dasjenige Älterer. Typischerweise leiden sie stärker unter der Trennung von ihrem gewohnten sozialen Umfeld und unter erzwungenem Alleinsein. In ihrer Persönlichkeit sind Jugendliche weniger verfestigt als Erwachsene, ihre Entwicklungsmöglichkeiten sind offener. Aus alledem ergeben sich spezielle Bedürfnisse, besondere Chancen und Gefahren für die weitere Entwicklung und eine besondere Haftempfindlichkeit, vor allem auch eine spezifische Empfindlichkeit für mögliche schädliche Auswirkungen des Strafvollzugs (vgl. Schaffstein/Beulke a. a. O., S. 6; Eisenberg, a. a. O., Einl. Rn. 5; J. Walter ZJJ 2003, S. 397)“ (Rn. 54 = NJW 2006, 2096).

„Ein der Achtung der Menschenwürde und dem Grundsatz der Verhältnismäßigkeit ... verpflichteter Strafvollzug muss diesen Besonderheiten, die jedenfalls bei einem noch jugendhaften Entwicklungsstand größtenteils auch auf Heranwachsende zutreffen, Rechnung tragen" (Rn. 56).

Ein spezieller und über das StVollzG hinausgehender Regelungsbedarf wird vom BVerfG insbesondere in Bezug auf Kontakte, körperliche Bewegung und die Art der Sanktionierung von Pflichtverstößen gesehen. „So müssen etwa die Besuchsmöglichkeiten für familiäre Kontakte - auch im Hinblick auf Art. 6 Abs. 2 GG-um ein Mehrfaches über denen im Erwachsenenvollzug (\$ 24 Abs. 1 S. 2 StVollzG) angesetzt werden. Erforderlich sind des weiteren gesetzliche Vorkehrungen dafür, dass innerhalb der Anstalt einerseits Kontakte, die positivem sozialen Lernen dienen können, aufgebaut und nicht unnötig beschränkt werden, andererseits aber die Gefangenen vor wechselseitigen Übergriffen geschützt sind. Nach derzeitigem Erkenntnisstand ist dazu die Unterbringung in kleineren Wohngruppen differenziert nach Alter, Strafzeit und Straftaten - etwa gesonderte Unterbringung von Gewalt- und Sexualtätern mit spezifischen Betreuungsmöglichkeiten - besonders geeignet" (Rn. 57).

Weiterhin wird besonderer Regelungsbedarf hinsichtlich des gerichtlichen Rechtsschutzes gesehen. Die derzeitige Regelung über $\$ \S 23$ ff. EGGVG genügt den Anforderungen des Art. 19 IV GG nicht (Stichwort: „effektiver Rechtsschutz"). Dementsprechend wird im Gegensatz zum derzeit gem. §§ 23 ff. EGGVG zuständigen OLG als Rechtsbehelfsinstanz ein ortsnahes, Möglichkeiten der mündlichen Kommunikation eröffnendes Rechtsmittelverfahren gefordert (vgl. BVerfG NJW 2006, 2096).

Zur Konkretisierung des Resozialisierungsvollzugs hat der Gesetzgeber zwar einen weiten Gestaltungsspielraum, jedoch „, hat er durch gesetzliche Festlegungen hinreichend konkretisierter Vorgaben Sorge zu tragen, dass für allgemein als erfolgsnotwendig anerkannte Vollzugsbedingungen und Maßnahmen die erforderliche Ausstattung mit den personellen und finanziellen Mitteln kontinuierlich gesichert ist. Der Staat muss den Strafvollzug so ausstatten, wie es zur
Realisierung des Vollzugsziels erforderlich ist (BVerfGE 35, 202, 235). Dies betrifft insbesondere die Bereitstellung ausreichender Bildungs- und Ausbildungsmöglichkeiten, Formen der Unterbringung und Betreuung, die soziales Lernen in der Gemeinschaft, aber auch den Schutz der Inhaftierten vor wechselseitiger Gewalt ermöglichen, ... ausreichende pädagogische und therapeutische Betreuung sowie eine mit angemessenen Hilfen für die Phase nach der Entlassung (vgl. BVerfGE 35, 202, 236) verzahnte Entlassungsvorbereitung. Bei den schulischen und beruflichen Ausbildungsangeboten ist darauf Bedacht zu nehmen, dass solche Angebote auch dann sinnvoll genutzt werden können, wenn wegen der Kürze der Haftzeit ein Abschluss während der Dauer der Haft nicht erreichbar ist" (Rn. 61 = NJW 2006, 2096 f.).

Des weiteren nimmt das BVerfG zur Bedeutung des empirischen Forschungsstandes bzw. Erfahrungswissens wie folgt Stellung: „Die gesetzlichen Vorgaben für die Ausgestaltung des Vollzugs müssen zudem auf sorgfältig ermittelten Annahmen und Prognosen über die Wirksamkeit unterschiedlicher Vollzugsgestaltungen und Behandlungsmaßnahmen beruhen (vgl. BVerfGE 106, 62, 152). Der Gesetzgeber muss vorhandene Erkenntnisquellen, zu denen auch das in der Vollzugspraxis verfügbare Erfahrungswissen gehört, ausschöpfen (vgl. BVerfGE 50, 290, 334) und sich am Stand der wissenschaftlichen Erkenntnis orientieren (vgl. BVerfGE 98, 169, 201)“ (Rn. $62=$ NJW 2006, 2097).

Obwohl internationale Mindestgrundsätze und Empfehlungen - soweit es sich nicht um in nationales Recht transformierte Konventionen o. ä. handelt (wie z. B. die EMRK) - keine Bindungswirkung für den deutschen Gesetzgeber haben, wertet das BVerfG ihre Bedeutung erheblich auf:

„Auf eine den grundrechtlichen Anforderungen nicht genügende Berücksichtigung vorhandener Erkenntnisse oder auf eine den grundrechtlichen Anforderungen nicht entsprechende Gewichtung der Belange der Inhaftierten kann es hindeuten, wenn völkerrechtliche Vorgaben oder internationale Standards mit Menschenrechtsbezug, wie sie in den im Rahmen der Vereinten Nationen oder von Organen des Europarates beschlossenen einschlägigen Richtlinien oder Empfehlungen enthalten sind ..., nicht beachtet beziehungsweise unterschritten werden (vgl. auch Schweizerisches Bundesgericht, Urteil vom 12. Februar 1992, BGE 118 Ia 64, 70)“ (Rn. 63).

Das BVerfG mahnt weiterhin eine zukunftsorientierte Erfolgskontrolle („Gesetzgeber ist zur Beobachtung" und ggf. „zur Nachbesserung" verpflichtet) und auf unterschiedliche Anstalten und Ausgestaltungen des Jugendvollzugs bezogene empirische Forschung, insbesondere Rückfallforschung an (vgl. Rn. 64).

Die Verfassungsbeschwerden hatten im Ergebnis dennoch keinen Erfolg. Das BVerfG gab dem Gesetzgeber vielmehr bis zur Verabschiedung eines verfassungsrechtlichen Ansprüchen genügen- den Gesetzes eine Übergangsfrist bis Ende 2007. Bis dahin sind Eingriffe in Grundrechte der Gefangenen in dem Maß hinzunehmen, wie dies „Zur Aufrechterhaltung eines ansonsten verfassungsgemäß geordneten Vollzuges unerlässlich ist" (Rn. 69 = NJW 2006, 2097).

\section{II.Die Bedeutung des Urteils für die anste- hende Jugendstrafvollzugsgesetzgebung}

Dass die derzeitige gesetzliche Grundlage des Jugendstrafvollzugs verfassungsrechtlichen Anforderungen nicht genügt und demgemäß ein detailliertes Gesetz zu schaffen ist, war nach der grundlegenden Entscheidung des BVerfG zum Erwachsenenstrafvollzug aus dem Jahr 1972 (vgl. BVerfGE 33, 1) allseits erwartet worden und nicht überraschend. Grundsätzlich waren bei den Vorüberlegungen in den letzten 25 Jahren dazu drei Optionen diskutiert worden (vgl. Dünkel 1990; 1992; 1999):

1. Eine Gesetzesregelung innerhalb des StVollzG (so jetzt der E-StVollzG Bayern),

2. eine Regelung innerhalb des JGG (ggf. mit ergänzenden umfangreicheren Verwaltungsvorschriften, vgl. hierzu auch § 115 JGG) oder

3. ein eigenständiges Jugendstrafvollzugsgesetz (so jetzt alle anderen vorliegenden Entwürfe des BJM, von Baden-Württemberg und der „Musterentwurf" von Tonford).

Der Verfasser dieses Beitrags hatte angesichts des geringen Innovationsgehalts des Entwurfs eines Jugendstrafvollzugsgesetzes des Bundesjustizministeriums von 1991 (der weitgehend identisch 1993 erneut vorgelegt wurde, vgl. i. e. Dünkel 1999) die Notwendigkeit eines eigenständigen Jugendstrafvollzugsgesetzes in Frage gestellt (vgl. Dünkel 1992). Die in den Vorentwürfen überzogene Technik der weitgehend wörtlichen Übernahme des StVollzG oder der Verweisung darauf ließ in der Tat die Besonderheiten des Jugendstrafvollzugs kaum erkennen. Im weiteren Verlauf der Arbeiten an einer gesetzlichen Regelung des Jugendstrafvollzugs seit 2001 wurde jedoch deutlich, dass es genügend Regelungsmaterie gibt, die ein eigenständiges JVollzG nicht nur rechtfertigt, sondern wegen der Andersartigkeit des Jugendvollzugs geradezu erfordert (vgl. Dünkel 2003; 2006, 561 ff.). Das BVerfG hat dies ausdrücklich bestätigt und festgestellt, dass eine schlichte Verweisung auf das StVollzG bzw. Übernahme der Vorschriften des StVollzG den Besonderheiten des Jugendstrafvollzugs nicht gerecht würde. Dementsprechend enthält selbst der bayerische Entwurf, der den Erwachsenen- und Jugendstrafvollzug in einem einheitlichen Gesetz regeln will (vgl. hierzu Ostendorf in diesem Heft), im Abschnitt für den Jugendstrafvollzug nicht weniger als 37 Artikel (Art. 119-156 EStVollzG Bayern).

Die Formulierungen des BVerfG in diesem Zusammenhang spiegeln das jugendkriminologi- 
sche Hintergrundwissen für die besonderen Lebenslagen und die besondere Haftempfindlichkeit junger Strafgefangener wider. Zutreffend wird auf die besonders einschneidende Wirkung des Freiheitsentzugs und das Zeitempfinden Jugendlicher hingewiesen, die typischerweise „unter der Trennung von ihrem gewohnten sozialen Umfeld und unter erzwungenem Alleinsein" leiden (BVerfG NJW 2006, 2096, 1. Sp. oben). Im Hinblick auf die Besonderheiten des Jugendalters wird demgemäß ein „spezieller Regelungsbedarf in Bezug auf Kontakte, körperliche Bewegung und die Art der Sanktionierung von Pflichtverstößen" gesehen und im Vergleich zum Erwachsenenvollzug mit Blick auf Art. 6 II GG „ein Mehrfaches“ an Besuchsmöglichkeiten gefordert (BVerfG a. a. O.). Alle vorliegenden Entwürfe (vgl. Bundesministerium der Justiz 2006; Bayerisches Ministerium der Justiz 2006; Justizministerium Baden-Württemberg 2006; Tondorf 2006) setzen diese Vorgabe mit mindesten vier Stunden Besuchszeit pro Monat (anstatt bisher einer Stunde) um. Der E-Tondorf sieht darüber hinausgehend noch Langzeitbesuche für Kinder und Ehegatten und Partner einer nichtehelichen Lebensgemeinschaft vor (vgl. § 21 II Nr. 1 E-Tondorf 2006). ${ }^{2}$ Da derartige erweiterte Besuchsformen im internationalen Vergleich immer häufiger zum Standard gehören, sehen die EPR 2006 in Nr. 24.4 vor, dass Besuche so organisiert werden sollen, dass sie dem Gefangenen ermöglichen, familiäre Beziehungen möglichst weitgehend (,in as normal a manner as possible $e^{\prime \prime}$ aufrecht zu erhalten. Ein Jugendstrafvollzugsgesetz sollte derartige (wenngleich etwas vage formulierte) Vorgaben aufgreifen (s. u.).

Die Bedeutung des BVerfG-Urteils geht jedoch weit über die Frage der Regelungsbedürftigkeit des Jugendstrafvollzugs an sich hinaus, denn das Urteil legt Maßstäbe bzw. Mindeststandards fest, die die anstehende Gesetzgebung der Länder zu beachten hat. Dies ist verdienstvoll, weil damit der eingangs zitierten Gefahr eines „Wettbewerbs der Schäbigkeit" zumindest in der Tendenz Argumente entgegengesetzt werden.

\section{Die Bedeutung internationaler Menschenrechtsstandards}

Wenn das BVerfG in diesem Zusammenhang die Bedeutung internationaler Regelungen des Europarats und der Vereinten Nationen hervorhebt und deren Unterschreitung als Indiz für eine den verfassungsrechtlichen Anforderungen nicht genügende Ausgestaltung des Jugendstrafvollzugs ansieht, so ergeben sich hieraus sehr weit reichende Konsequenzen für die anstehende Gesetzgebung. Bemerkenswert erscheint hierbei, dass auch sog. Soft-Law von Empfehlungen des Europarats und der Vereinten Nationen zu beachten sind, die formell keine Bindungswirkung haben. Als „Messlatte" sind hierbei einerseits die aktuellen European Prison Rules (EPR) von 2006 (vgl. Council of Europe 2006), zum anderen die Mindestgrundsätze der Vereinten Nationen zum Schutz von Jugend- lichen unter Freiheitsentzug („United Nations Rules for the Protection of Juveniles deprived of their Liberty", im Folgenden UN-Rules) von 1990 heranzuziehen (deutsche Übersetzung bei Höynck/Neubacher/Schüler-Springorum 2001, 94 ff.).

So dürfen z. B. finanzielle Überlegungen („die Mittelknappheit") nach Nr. 4 der EPR keine Rechtfertigung für menschenrechtswidrige Unterbringungsformen sein. Dies bedeutet, dass beispielsweise auch die nur vorübergehende Überbelegung und im Gefolge davon gemeinschaftliche Unterbringung, „wenn die räumlichen Verhältnisse dies erfordern" - wie es noch in § $2001 \mathrm{Nr}$. 3 StVollzG vorgesehen ist - im Jugendstrafvollzug nicht möglich wäre. Der Gesetzgeber ist im Übrigen schon wegen Nr. 18.5 der EPR zur Einzelunterbringung während der Ruhezeit grundsätzlich verpflichtet (die EPR sehen Ausnahmen davon nur vor, wenn die gemeinschaftliche Unterbringung aus Gründen des Schutzes für den Gefangenen, z. B. bei Selbstmordgefährdung, angezeigt erscheint). Alle Hafträume, insbesondere die für die nächtliche Unterbringung vorgesehenen Räume, sind menschenwürdig auszugestalten und die Intimsphäre ist so weit möglich zu schützen (Nr. 18.1 EPR). Die bisher vorliegenden Entwürfe des BJM, von Bayern und Baden-Württemberg haben diese Vorgabe leider nicht in konkrete Vorgaben umgesetzt. Insoweit vorzugswürdig ist ein von Tondorf vorgelegter "Musterentwurf“, der sehr viel konkreter beispielsweise eine Mindestbodenfläche von 10 qm für eine Einzelzelle fordert (§ 40 VIII Nr. 2 E-Tondorf, vgl. Tondorf 2006). Dass Hafträume gut belüftbar, genügend beheizt und mit ausreichender Fensterfläche ausgestattet sein müssen (die z. B. Lesen bei Tageslicht ermöglichen), gilt als internationaler Standard (vgl. Nr. 18.1, 18.2.a EPR 2006). Die EPR fordern in Nr. 18.3, dass die nationale Gesetzgebung diesbezüglich konkrete Mindestfestlegungen treffen soll. Auch insoweit ist der Musterentwurf von Tondorf konkreter, indem er $z$. B. Fensterflächen von mindestens $1 / 8$ bis $1 / 7$ der Bodenfläche fordert ( $\$ 40$ VIII Nr. 2 ETondorf unter Bezugnahme auf AK-StVollzGHuchting/Lehmann § 144 Rn. 6).

Als weitere Vorgaben der EPR sind in diesem Zusammenhang beispielhaft zu nennen:

Die EPR (vgl. den Grundsatz Nr. 9 und Nr. 92 und 93 EPR) ebenso wie die UN-Rules für junge Inhaftierte (vgl. Nr. 72-78) fordern neben staatlichen Aufsichtsbehörden die Einrichtung unabhängiger Inspektionsorgane. Die UN-Rules schlagen in Nr. 77) unabhängige Ombudsleute vor. Dieser Gedanke (vgl. hierzu Vorschläge bei Dünkel 1999, 129 ff.; 2006, 562) wird bislang nur im Entwurf von Tonford ansatzweise aufgegriffen, hier allerdings als Organ der informellen Konfliktschlichtung, nicht als vom Einzelfall losgelöstes Inspektionsgremium (vgl. § 34 E-Tondorf 2006).

Der Vorrang informeller Konfliktregelung, wie ihn insbesondere auch der E-BJM 2006 in $\$ 33$ verankert hat, wird in Nr. 56.1 und 56. 2 der EPR betont („Disciplinary procedures shall be mechanisms of last resort" , "Wherever possible, prison authorities shall use mechanisms of restoration and mediation to resolve disputes with and among prisoners").

Eine weitere Vorgabe machen die EPR hinsichtlich der Anlasstaten für disziplinarische Sanktionen. Generalklauseln wie „Verstoß gegen Pflichten" (die im Gesetz nicht eindeutig definiert werden) sind danach nicht zulässig. Vielmehr muss nach Nr. 57.2 das Gesetz eindeutig das einen Disziplinartatbestand betreffende Verhalten, das Verfahren und die vorgesehenen Sanktionen beschreiben. Die Verhältnismäßigkeit der Sanktionierung wird in Nr. 60.2 besonders hervorgehoben. Der E-BJM 2006 hat diesen Vorgaben in § 34 Rechnung getragen. Gleichwohl ist die Kritik, Entweichungsversuche entgegen einer verbreiteten Auffassung zum StVollzG für Erwachsene zu pönalisieren, ebenso wie die an der vielleicht zu weit gefassten Generalklausel des $\S 34$ Nr. 8 berechtigt. $^{3}$

Dass der Rechtsschutz gegen Maßnahmen der Anstalt bislang im Jugendstrafvollzug völlig unzureichend ist, hat das BVerfG zu Recht angemahnt (NJW 2006, 2096). Die EPR legen in Nr. 59 detailliert rechtsstaatliche Mindeststandards fest. Die weitgehende Verweisung auf das StVollzG in $\S 35$ des E-BJM 2006 genügt diesen internationalen Vorgaben insofern nicht, als die EPR grundsätzlich von einer mündlichen Verhandlung ausgehen, im Rahmen derer der Beschuldigte die Vernehmung von Zeugen beantragen kann, ihm falls notwendig ein kostenloser Dolmetscher bzw. Anwalt zur Seite gestellt werden muss etc. (vgl. Nr. 59.c.-e.). Das deutsche Antragsverfahren nach $\S 109 \mathrm{ff}$. StVollzG ist grundsätzlich schriftlich und es gilt das Freibeweisverfahren. Ferner wird ein Rechtsbeistand nur nach den restriktiven Regelungen der ZPO gewährt (vgl. § 120 II StVollzG, auf den § 35 II des E-BMJ 2006 verweist). Die EPR sind insofern strenger und sollten Anlass dafür geben, das Disziplinarverfahren, auch wenn es nur „ultima ratio“ sein soll, jugendgemäßer auszugestalten. Schon deshalb spricht manches dafür, das Rechtsbehelfsverfahren beim ortsnahen Jugendrichter als Vollstreckungsleiter (so § 35 II ETondorf 2006) anstatt bei der Jugendkammer des LG anzusiedeln (so aber § 35 IV E-BJM 2006).

\section{Die Berücksichtigung des Stands der Behandlungsforschung und Grenzen des gesetzgeberischen Gestaltungs- spielraums}

Das BVerfG hat immer schon Zurückhaltung geübt, wenn es um Festlegungen im Hinblick auf bestimmte Formen der Vollzugsgestaltung bzw. Resozialisierung geht und insoweit einen weiten gesetzgeberischen Gestaltungsspielraum ange- 
nommen (BVerfG NJW 2006, 2096 m. w. N., s. o.; kritisch hierzu Ostendorf NJW 2006, 2074). Dies lag zumindest für die 1970er und 1980er Jahre deshalb nahe, weil die Frage der Wirksamkeit der Straftäterbehandlung umstritten und z. T. offen war. Inzwischen ist unter Ablehnung der „Nothing works"-Doktrin der 1970er Jahre einhellige Meinung geworden, dass der Slogan „something works" den Forschungsstand besser beschreibt. Es ist den amerikanischen und deutschen Meta-Analysen zur Behandlungsforschung zu verdanken, dass wir heute über relativ gut gesichertes Wissen verfügen, „was wirkt“ („what works“; vgl. Lösel 1993; 2001; Dünkel/Drenkhahn 2001; McGuire 2003; Sherman u. a. 2006).

In der vorliegenden Entscheidung nimmt das BVerfG diese Beliebigkeit der Ausgestaltung jedoch erstmals deutlich zurück, indem es „allgemein als erfolgsnotwendig anerkannte Vollzugsbedingungen und Maßnahmen" benennt. Dazu gehören „die Bereitstellung ausreichender Bildungs- und Ausbildungsmöglichkeiten, Formen der Unterbringung und Betreuung, die soziales Lernen in Gemeinschaft, aber auch den Schutz der Inhaftierten vor wechselseitiger Gewalt ermöglichen" (BVerfG NJW 2006, 2096, r. Sp. unten). Dies hat der Entwurf des BJM zutreffend in der geforderten grundsätzlichen Einzelunterbringung während der Ruhezeit im Rahmen eines Wohngruppenvollzugs umgesetzt (vgl. § 19 E-BJM 2006). Auch die Forderung, dass zwei Drittel der Haftplätze für allgemeine und berufliche Ausbildungszwecke vorzuhalten sind (vgl. § 40 VII EBJM 2006), wird dem verfassungsrechtlichen Anspruch gerecht.

Die erkennbar wesentlichsten Unterschiede zwischen den bisher vorliegenden Entwürfen liegen im Bereich der Öffnung des Vollzugs. Während der BJM-Entwurf und der Entwurf Tondorf am Vorrang des offenen Vollzugs und einem System der überleitungsorientierten Vollzugsgestaltung durch Hafturlaub und Lockerungen festhalten, sind Bayern und Baden-Württemberg hier sehr viel restriktiver, was bereits durch die programmatische Orientierung an Sicherheit bzw. dem Schutz der Allgemeinheit zum Ausdruck gelangt (zur Kritik vgl. auch Ostendorf in diesem Heft). Abgesehen davon, dass auch das BVerfG die darin deutlich werdende unsinnige Polarisierung von Resozialisierung und Schutz der Allgemeinheit für obsolet erklärt, weil ein richtig verstandener Schutz der Allgemeinheit keinen Gegensatz „zum Integrationsziel des Vollzugs" darstellt (vgl. BVerfG NJW 2006, 2095, r. Sp. Mitte), ${ }^{4}$ ergibt sich aus der Sicht der Behandlungsforschung die Frage, ob die Öffnung bzw. Lockerung des Vollzugs nicht ein erfahrungswissenschaftlich („evidence based“) gut begründetes Prinzip ist. Zu den wissenschaftlich belegten Leitlinien erfolgreicher Straftäterbehandlung gehören Formen der Vollzugsgestaltung, die soziale Kompetenzen fördern bzw. erhalten. Dass von einem systematischen Konzept der Öffnung des Vollzugs mit überleitungsorientier- ten Entlassungshilfen (Lockerungen, Hafturlaub, offener Vollzug bis hin zum Freigang) ein signifikanter Beitrag der Rückfallreduzierung erwartet werden darf, belegen die in den letzten Jahren durch verschiedene Meta-Analysen dokumentierten Strategien erfolgreicher Straftäterbehandlung. ${ }^{5}$ Eine der erfolgreichen Strategien betrifft Formen des sozialen Trainings mit gut strukturierten Abläufen ${ }^{6}$ wie sie im Rahmen einer gezielten Öffnung des Vollzugs gut umgesetzt werden kann. Die Vermittlung beruflicher Fertigkeiten sowie sozialer Handlungs- und Problemlösungskompetenz setzt die Öffnung des Vollzugs und verhaltenstherapeutisch strukturierte Lern- und Trainingsfelder voraus. Auch die Gemeindeorientierung wird explizit als Erfolg versprechende Strategie genannt. ${ }^{7}$

Dass dies u. a. im offenen, insbesondere Freigänger-Vollzug gut gelingen kann, dürfte nicht umstritten sein. Zwar kann man einzelne Lockerungsmaßnahmen isoliert in ihrem Potenzial der Rückfallvermeidung nicht abschätzen, jedoch ist ein insgesamt überleitungsorientierter Vollzug mit einem ganzen Bündel von Entlassungsvorbereitungsmaßnahmen, zu denen als wesentliches Element im Sinn von Belastungsproben ${ }^{8}$ Vollzugslockerungen und Hafturlaub gehören, unzweifelhaft als resozialisierungsfördernder im Vergleich zu einer unvorbereiteten Entlassung aus dem geschlossenen Verwahrvollzug anzusehen. Obwohl kausale Erklärungen für die empirisch immer wieder bestätigte geringere Rückfälligkeit von Entlassenen des offenen und Freigängervollzugs (vgl. Dünkel 2004, 106 f. m. w. N.) schwierig sind, darf ein Rückfall mindernder Beitrag offener Vollzugsformen einschließlich Lockerungen dann angenommen werden, wenn diese unter Beachtung weiterer Prinzipien erfolgreicher Straftäterbehandlung (wie z. B. das Risk-, Needs- oder ResponsivityPrinzip) eingesetzt werden (vgl. i. E. Andrews u. a. 1990; Lösel 2001; Dünkel/Drenkhahn 2001; Dünkel 2004).

Diese Maßnahmen zu diskreditieren, womit sich insbesondere der ehemalige Hamburger Justizsenator Kusch profilierte, und - wie aus Hamburg zu lesen ist - weitgehend abzuschaffen (vgl. Pressemitteilung des Hamburger Justizsenats, FR v. 24.7.2006, S. 4), ist verfassungsrechtlich nicht haltbar, weil damit anerkanntes wissenschaftliches und praktisches Erfahrungswissen missachtet wird. Dementsprechend fordert das BVerfG zu Recht „eine mit angemessenen Hilfen für die Phase nach der Entlassung verzahnte Entlassungsvorbereitung". Darunter fällt u. a. die Einbeziehung von Externen (vgl. \$§ 88 VI, 89 JGG im E-BJM 2006, der die Einbeziehung der Bewährungshilfe und freien Straffälligenhilfe in die Entlassungsvorbereitung der Anstalt mindestens drei Monate vor der Entlassung vorsieht). ${ }^{9}$ Dementsprechend sehen die EPR 2006 in Nr. 107.1-107.5 ein differenziertes System der Entlassungsvorbereitung und eines abgestuften Übergangs in die Freiheit in Zusammenarbeit mit den Einrichtungen der Nachentlassungshilfe vor.
Ob die derzeit schon bestehenden gravierenden Unterschiede der Vollzugsgestaltung in diesem Bereich ${ }^{10}$ auch ein verfassungsrechtliches Problem darstellen, wie dies insbesondere Seebode in der Anhörung des Bundestags und Bundesrats zur Föderalismusreform im Hinblick auf Art. 102 II GG (Bestimmtheitsgebot) bejaht hat, muss derzeit noch offen bleiben. Immerhin hat das BVerfG zur regional unterschiedlichen Vollzugspraxis (wie sie insbesondere im Gutachten des Verf. im vorliegenden Verfahren deutlich gemacht wurden, vgl. BVerfG a. a. O., Rn. 22-26) indirekt Kritik anklingen lassen und für die Zukunft die Erhebung vergleichbarer Daten gefordert, die bis zur Anstaltsebene eine Erfassung unterschiedlicher Vollzugsformen und darauf fußend die Beurteilung deren Wirksamkeit ermöglichen müsse (BVerfG NJW 2006, 2097, 1. Sp. unten).

\section{Ausblick}

Das Urteil des BVerfG ist in jeder Hinsicht verdienstvoll und weiterführend für die anstehende Gesetzgebung zum Jugendstrafvollzug. Es gibt Hoffnung, dass die Reformanstrengungen sich nicht im „Wettbewerb der Schäbigkeit" für einen möglichst kostengünstigen Vollzug erschöpfen werden, sondern der fachliche Diskurs um den bestmöglichen Resozialisierungsvollzug und die Beachtung der Menschenrechte gehen wird. Dass dabei wesentlich auf empirisches und praktisches Erfahrungswissen abzustellen ist, wird hoffentlich die Strafvollzugsforschung weiter anregen. Ideologisch begründete Glaubenskämpfe und populistische Strafvollzugspolitik können am ehesten durch eine Orientierung an den inzwischen empirisch gut belegten Prinzipien erfolgreicher Straftäterbehandlung überwunden werden. Auch insoweit hat das BVerfG verdienstvoll dazu beigetragen, dass sich die Debatte versachlichen kann. Nicht umsonst hebt $§ 39$ E-BJM ${ }^{11}$ die Forschung der kriminologischen Dienste ebenso wie unabhängiger universitärer und anderer Institutionen hervor. Die Länder (und Forschungsfördereinrichtungen) sollten dafür die notwendigen sachlichen und personellen Mittel bereitstellen.

Andererseits werden einzelne Länder wohl kaum von einer programmatischen Vorrangstellung der Sicherheit abzubringen sein, die jedoch letztlich nur die ohnehin (z. T. seit langem) bestehende restriktive Praxis der Öffnung des Vollzugs (vgl. Dünkel/Schüler-Springorum 2006) „legitimieren" soll. Hiergegen werden argumentativ nur die auch in Bayern oder Hamburg irgendwann empirisch belegbaren Defizite helfen. Andererseits gibt es berechtigte Hoffnung, dass sich eine Mehrheit der Bundesländer auf ein vernünftiges Gesetz, wie es durch den Entwurf des BJM von 2006 vorgezeichnet wurde, verständigen wird. Selbst die Entwürfe von Bayern und Baden-Württemberg haben zu etwa 90\% oder mehr den Vorschlag des Entwurfs des BJM übernommen. Der Unsinn der beschlossenen Föderalismusreform in diesem Bereich könnte damit kaum besser bestätigt werden. 


\section{Literatur:}

AK-StVollzG (2006): Kommentar zum Strafvollzugsgesetz (AK-StVollzG). Hrsg. v. J. Feest. 5. Aufl., Neuwied, Kriftel: Luchterhand.

Andrews, D. A., u. a. (1990): Does Correctional Treatment work? A Clinically Relevant and Psychologically Informed Metaanalysis. Criminology 28, S. 369-404 .

Bayerisches Staatsministerium der Justiz (2006): Gesetz über den Vollzug der Freiheitsstrafe, der Jugendstrafe und der Sicherungsverwahrung. Bayerisches Strafvollzugsgesetz - BayStVollzG. Diskussionsentwurf. Stand: 3. August 2006. München: Bayerisches Staatsministerium der Justiz (als pdf-Datei im Internet unter: http://www.justiz.bayern.de/imperia/md/content/stmj _internet/ministerium/ministerium/gesetzgebung/gesetzentwurf_baystvollzg.pdf, zitiert: E-StVollzG Bayern)

Bundesministerium der Justiz (2006): Entwurf eines Gesetzes zur Regelung des Jugendstrafvollzuges (GJVollz). Stand: 7. Juni 2006. Berlin: Bundesministerium der Justiz (im Internet als pdf-Datei unter http://www.bmj.de/media/archive/1237.pdf, zitiert: E-BJM)

Council of Europe (2006): Recommendation Rec (2006) 2 of the Committee of Ministers to member states on the European Prison Rules. Strasbourg: Council of Europe (im Internet unter https://wcd.coe.int/ ViewDoc.jsp?id=955747\&BackColorInternet=9999CC\&Bac kColorIntranet=FFBB55\&BackColorLogged=FFAC75, zitiert: EPR)

Dünkel, F. (1990): Freiheitsentzug für junge Rechtsbrecher. Bonn: Forum Verlag Godesberg.

Dünkel, F. (1992): Brauchen wir ein Jugendstrafvollzugsgesetz? ZRP 25, S. 176-181.

Dünkel, F. (1996): Empirische Forschung im Strafvollzug. Bonn: Forum Verlag Godesberg.

Dünkel, F. (1999): Jugendstrafvollzug zwischen Erziehung und Strafe - Entwicklungen und Perspektiven im internationalen Vergleich. In: Feuerhelm, W., Schwind, H.-D., Bock, M. (Hrsg.): Festschrift für Alexander Böhm. Berlin, New York: Walter de Gruyter, S. 99-140.

Dünkel, F. (2003): Situation und Reform des Jugendstrafvollzugs in Deutschland. RdJB 51, S. 318-334.

Dünkel, F. (2004): Die Öffnung des Vollzugs und Vollzugslockerungen als Sicherheitsrisiko? In: Strafverteidigervereinigungen (Hrsg.): Internationalisierung des Strafrechts. Fortschritt oder Verlust an Rechtsstaatlichkeit? 27. Strafverteidigertag, Dresden, 14.-16. März 2003. Berlin: Schriftenreihe der Strafverteidigervereinigungen, S. 89-129.

Dünkel, F. (2006): Die Reform des Jugendstrafvollzugs in Deutschland. In: Feltes, T., Pfeiffer, C., Steinhilper (Hrsg.): Kriminalpolitik und ihre wissenschaftlichen Grundlagen. Festschrift für Prof. Dr. Hans-Dieter Schwind zum 70. Geburtstag. Heidelberg: C. F. Müller Verlag 2006, S. 549-570.

Dünkel, F., Drenkhahn, K. (2001): Behandlung im Strafvollzug: von "nothing works" zu "something works". In: Bereswill, M., Greve, W. (Hrsg.): Forschungsthema Strafvollzug. Baden-Baden: Nomos, S. 387-417.

Dünkel, F., Schüler-Springorum, H. (2006): Strafvollzug als Ländersache? Der "Wettbewerb der Schäbigkeit" ist schon im Gange! ZfStrVo 55, S. 145-149.

Höynck, T., Neubacher, F., Schüler-Springorum, H. (2001): Internationale Menschenrechtsstandards und das Jugendkriminalrecht. Berlin: Forum Verlag Godesberg.

Justizministerium Baden-Württemberg (2006): Entwurf eines Jugendstrafvollzugsgesetzes (JStVollzG, E-BW). Stuttgart: Justizministerium Baden-Württemberg (im Internat als pdf-Datei unter: http://www.jum.baden-
wuerttemberg.de/servlet/PB/show/1200200/ E-JStVollzG-BW\%20Stand\%2007062006.pdf, zitiert: E-BW).

Lipton, D. S. (1998): The Effectiveness of Correctional Treatment Revisited Thirty Years Later: Preliminary Meta-Analytic Findings from the CDATE study. Vortrag beim 12. Internationalen Kongress für Kriminologie, Seoul, 24.-29.8.1998 (Bezug über doug.lipton@earthlink.org).

Lösel, F. (1993): Sprechen Evaluationsergebnisse von Meta-Analysen für einen frischen Wind in der Straftäterbehandlung? In: Egg, R. (Hrsg.), Sozialtherapie in den 90er Jahren, Wiesbaden: KrimZ, S. 21-31.

Lösel, F. (2001): Behandlung oder Verwahrung? Ergebnisse und Perspektiven der Interventionen bei „psychopathischen“ Straftätern. In: Rehn, G., u. a. (Hrsg.): Behandlung „gefährlicher Straftäter". Herbolzheim: Centaurus, S. 36-53.

McGuire, J. (2003) (Hrsg.): Offender Rehabilitation and Treatment. Effective Programmes and Policies to Reduce Re-offending. Chichester: John Whiley \& Sons.

Sherman, L. W., Farrington, D. P., Welsh, B. C., MacKenzie, D. L. (2006) (Hrsg.): Evidence-Based Crime Prevention. Revised edition. London, New York: Routledge.

Tondorf, G. (2006): Musterentwurf zu einem Jugendstrafvollzugsgesetz der Länder. Unveröff. Ms. Köln 2006 (zitiert: E-Tondorf).

Vennard, J., Hedderman, C. (1998): Effective interventions with offenders. In: Goldblatt, P., Lewis, C. (Hrsg.): Reducing offending: an assessment of research evidence on ways of dealing with offending behaviour. London: Home Office, S. 101-119.

Prof. Dr. Frieder Dünkel, Lehrstuhl für Kriminologie, lehrt an der Universität Greifswald und ist Mitherausgeber dieser Zeitschrift

\section{Fußnoten}

1 Die Angabe der Rn. beziehen sich auf die Internetveröffentlichung des Volltextes des Urteils unter www.bundesverfassungsgericht.de/entscheidungen/ rs20060531_2bvr167304.html.

2 Die E-BW und E-StVollzG Bayern sehen diese Erweiterung nur für Kinder, nicht aber die Lebenspartner der jungen Gefangenen vor.

3 Vgl. schon Dünkel 2006, 567; ebenso Tonford 2006; nicht zuzustimmen ist Tonford in der Kritik an der isolierenden Einzelhaft in der „Verpackung" als be- sondere Sicherungsmaßnahme gem. § $31 \mathrm{~V}$ E-BJM 2006. Der E-BJM will den Arrest als Disziplinarmaßnahme (im Gegensatz zu den Entwürfen von BadenWürttemberg und Bayern) ersatzlos streichen. Dass in Fällen akuter Selbstmordgefährdung oder der Gefährdung anderer Gefangener (gewalttätige) Inhaftierte kurzfristig isoliert werden können müssen, erscheint außer Frage, denn der Staat ist - wie das BVerfG betont (NJW 2006, 2096, l. Sp.) zum Schutz vor wechselseitigen Übergriffen der Gefangenen verpflichtet. Sicherungs- und Disziplinarmaßnahmen haben unterschiedliche Anlässe und Zielsetzungen. Dass in der Praxis Sicherungsmaßnahmen gelegentlich als versteckte Disziplinierung missbraucht werden, ist bekannt (vgl. schon Dünkel 1996, 20, 101) und durch verschiedene Kontrollmechanismen zu unterbinden. Ein verbessertes Rechtsmittelsystem, die Einbeziehung von Ombudsleuten und unabhängige Inspektionen könnten insoweit präventiv wirken.

4 Die European Prison Rules stellen dementsprechend beim Ziel des Strafvollzugs für verurteilte Straftäter einzig und allein auf das Resozialisierungsziel ab, vgl. Nr. 102.1 EPR. Im Übrigen wird - wie schon 1987 betont, dass das Wesen der Freiheitsstrafe einzig im Entzug der Bewegungsfreiheit besteht und dementsprechend die Vollzugsgestaltung das dem Freiheitsentzug immanente Leiden nicht verstärken darf, vgl. Nr. 102.2 EPR. Damit wird auch aus europäischer Sicht einer schuldbezogenen Vollzugsdifferenzierung (z. B. Verweigerung von Lockerungen wegen der Schwere der Straftat) der Boden entzogen (so inzwischen auch OLG Frankfurt NStZ 2002, 53), vgl. hierzu Dünkel/Morgenstern/Zolondek in diesem Heft.

5 Vgl. zusammenfassend Lösel 1993; Lipton 1998; Vennard/Hedderman 1998; Dünkel/Drenkhahn 2001; Sherman u. a. 2006.

6 Vgl. hierzu insbesondere Andrews u. a. 1990

7 Vgl. zusammenfassend Dünkel/Drenkhahn 2001.

8 die nach st. Rspr. des BVerfG eine erfahrungswissenschaftlich gestützte Prognosestellung im Hinblick auf $\S \S 57,57$ a StGB häufig erst ermöglichen.

$9 \mathrm{Zu}$ weitergehenden Forderungen (mindestens 6 Monate vor der Entlassung) vgl. Dünkel 2006, $566 \mathrm{f}$.

$10 \mathrm{Vgl}$. zur immer schon restriktiven Lockerungspraxis in Bayern im Vergleich zu Berlin oder NordrheinWestfalen Dünkel 2004; zum drastischen Abbau des offenen Vollzugs in Hamburg und Hessen (im Gegensatz zu Berlin und Nordrhein-Westfalen) vgl. Dünkel/Schüler-Springorum 2006, $145 \mathrm{ff}$.

11 Ebenso § 39 E-Tondorf
Mit der Föderalismusreform wird es neue Debatten um den Strafvollzug geben. Erfahrungsgemäß wirken praktisches Erfahrungswissen und Empirie auf die Dauer versachlichend. Vielleicht kann die europäische Strafvollzugsforschung ideologisch begründete Glaubenskämpfe und populistische Strafvollzugspolitik bremsen und inzwischen empirisch gut belegte Prinzipien erfolgreicher Straftäterbehandlung europaweit durchsetzen. Dies könnte das politische Problem, in allen Bundesländern Mehrheiten für angemessene Strafvollzugsgesetze zu organisieren, erleichtern. Aber dies setzt voraus, dass alle Informierten sich artikulieren. 